\title{
Spectroscopic Evidence for Room Temperature Interaction of Molecular Oxygen with Cobalt Porphyrin Linker Sites within a Metal-Organic Framework
}

\author{
Nicole Lahanas, Pavel Kucheryavy, Jenny V. Lockard* \\ Department of Chemistry, Rutgers University-Newark \\ Newark, New Jersey 07102, United States
}

Supporting information

Materials: All starting materials for synthesis were purchased from TCI America or Alfa Aesar. The CoTPP porphyrin reference complex was obtained from Frontier Scientific.

\section{Synthesis:}

Co-PCN222: A double scale reaction was carried out compared to the literature procedure.[1] Yield: 139 mg, 63\%. Analysis: XRD (Figure S1), IR (Figure S2): 3353 (w), 2976 (w), 2924 (w), 1697 (w), 1604 (s), 1552 (s), 1415 (s), $1351(\mathrm{~m}), 1305$ (w), 1209 (m), 1178 (m), 1099 (m), 1007 
(s), $866(\mathrm{~m}), 793(\mathrm{~s}), 765(\mathrm{~s}), 725(\mathrm{~m}) \mathrm{cm}^{-1}$. UV-VIS diffuse reflectance $\lambda$, nm: 422, 542, 576

(sh). Activated sample was prepared by soaking the as synthesized Co-PCN-222 in acetone followed by heating in the vacuum at $120{ }^{\circ} \mathrm{C}$ for $12 \mathrm{~h}$ in a Schlenk tube.

\section{Characterization:}

Powder X-ray diffraction (XRD) patterns were recorded using Bruker APEX II equipped with a 4K CCD detector and $\mathrm{Cu}$ X-ray tube. Full metallation of the metalloporphyrin precursors was confirmed by high resolution ESI-MS using a Bruker Daltonics Apex-ultra 70 hybrid Fourier transform mass spectrometer and UV-vis spectroscopy. Diffuse reflectance UV-vis spectra were collected using a Cary-Varian UV-visible-NIR spectrophotometer equipped with a diffuse reflectance accessory (Harrick Scientific). Samples were diluted and finely ground with $\mathrm{KBr}$. Final spectra were plotted in Kubelka-Munk units.

\section{In situ diffuse reflectance measurements:}

In situ diffuse reflectance experiments were carried out on the UV-vis setup described above using a controlled environment reaction chamber (Harrick Scientific). KBr-diluted Co-PCN-222 sample was heated to $120{ }^{\circ} \mathrm{C}$ under dynamic vacuum and held under these conditions for 6 hours. The activation process was monitored by DR spectroscopy. Once no additional spectral changes were observed, the sample was cooled to room temperature for subsequent gas treatment. Spectra were recorded at room temperature in the vacuum, and upon exposure to 1 atm of either nitrogen or oxygen gas environment. Spectra were again recorded 15 minutes after gas treatments. 


\section{X-ray absorption spectroscopy:}

X-ray absorption data were collected at the Co K-edge $(7709 \mathrm{eV})$ in transmission mode at Beamline 2-2 at SSRL. The X-ray white beam was monochromatized by a $\operatorname{Si}(111)$ monochromator and detuned by $30 \%$ to minimize the harmonic content of the beam. Cobalt metal foil was used as reference for energy calibration. The incident beam intensity $\left(I_{0}\right)$ was measured by a $15 \mathrm{~cm}$ ionization chamber with $100 \% \mathrm{~N}_{2}$ for both metal K-edges. The transmitted $\left(I_{t}\right)$ and reference $\left(I_{r}\right)$ beam intensities were both measured using $30 \mathrm{~cm}$ ionization chambers, each with a $75 \% \mathrm{~N}_{2}$ and $25 \% \mathrm{Ar}$ gas mixture. A mixture of approximately $20 \mathrm{mg}$ of the CoTPP reference complex thoroughly ground with $\sim 100 \mathrm{mg}$ of boron nitride was packed and sealed in 3 mm Kapton tubes to yield approximately one X-ray absorption length. MOF samples were ground and packed in the same $3 \mathrm{~mm}$ sample tube format without any dilution. The activated MOF sample was packed and sealed inside a glovebox. For the oxygen loaded MOF sample, CoPCN222- $\mathrm{O}_{2}$, a portion of the activated MOF sample was packed in a 3-mm sample tube and removed from the glovebox in the schlenk flask and exposed to $100 \%$ oxygen gas environment for three days. The sample was subsequently sealed inside the tube prior to removal from the schlenk line. 


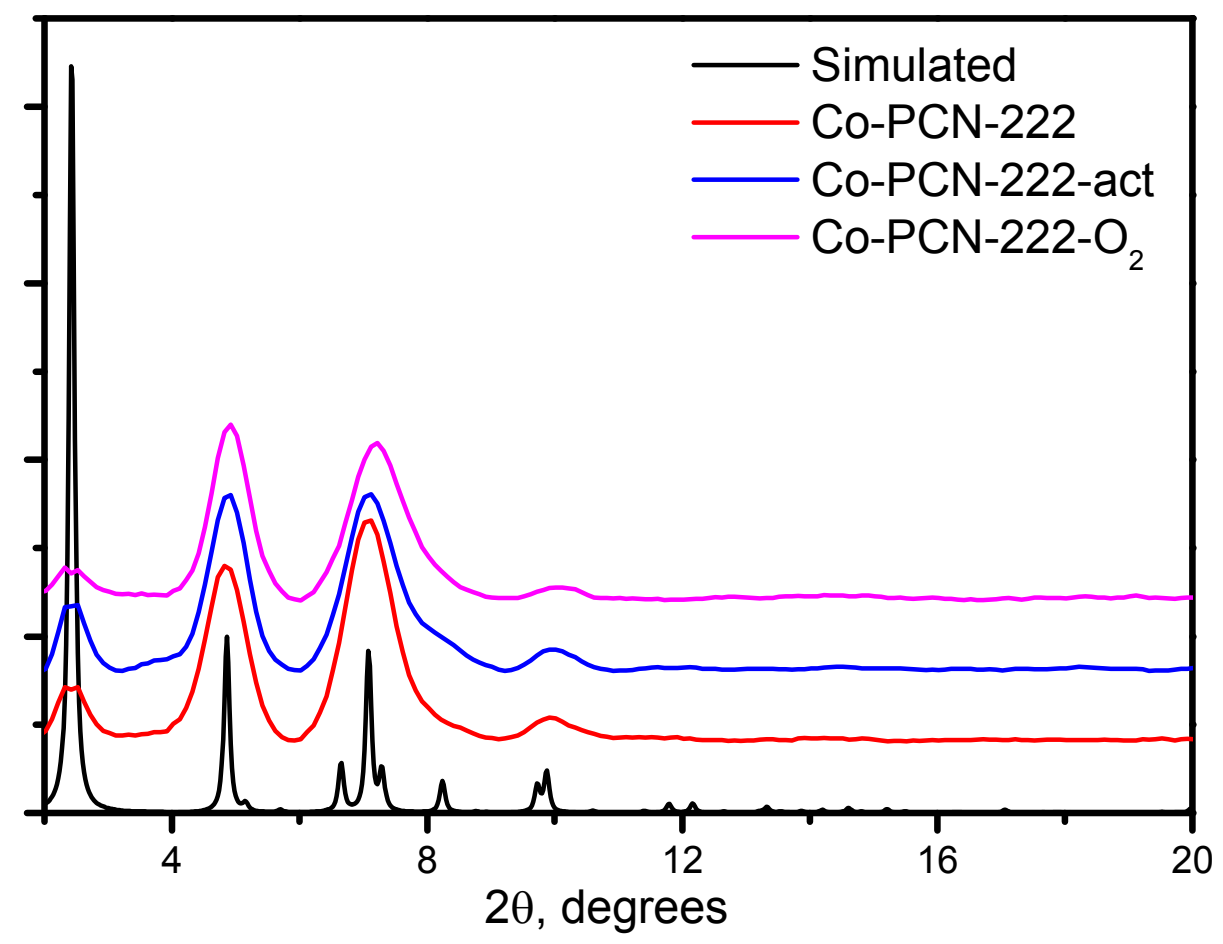

Figure S1. XRD pattern for Co-PCN222

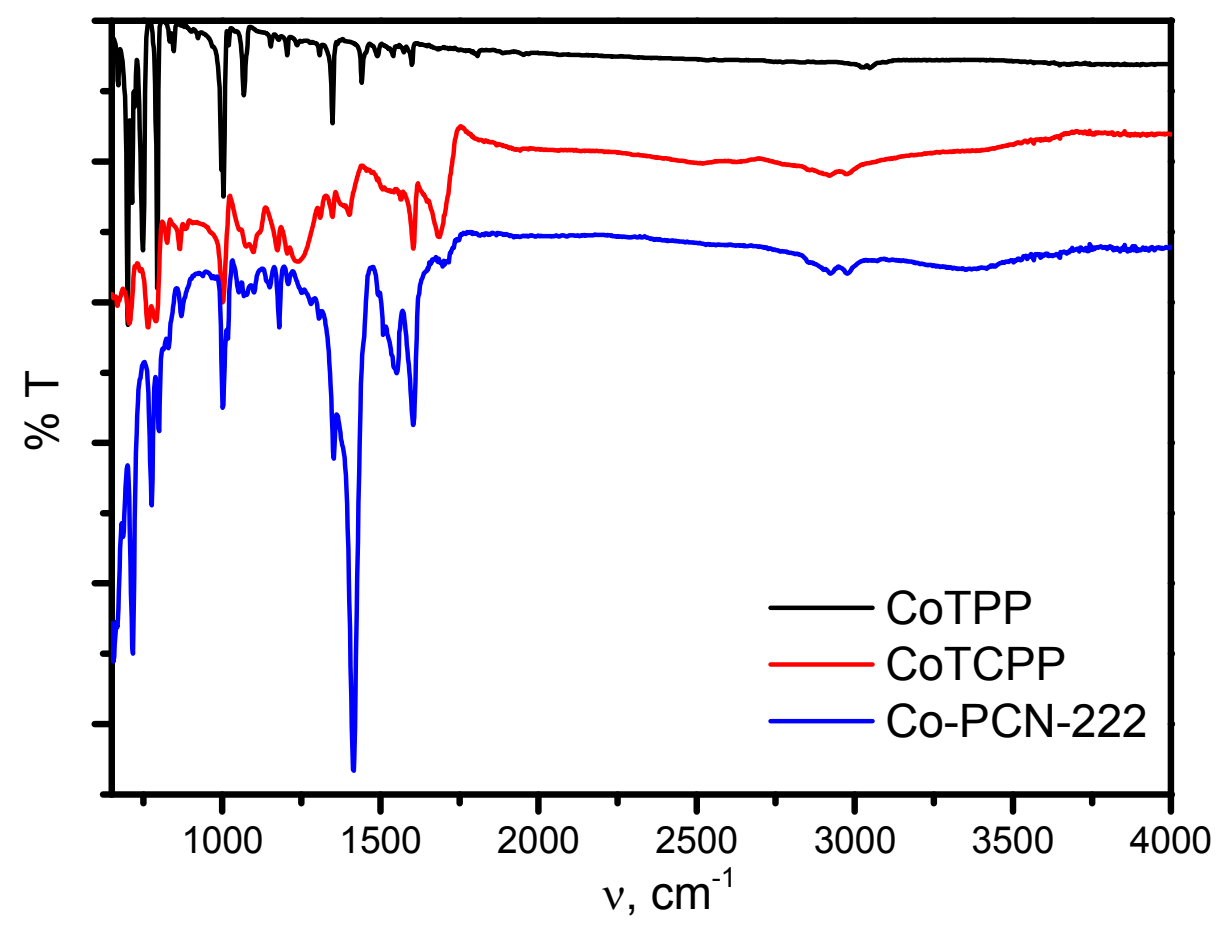

Figure S2. IR spectrum of Co-PCN222 


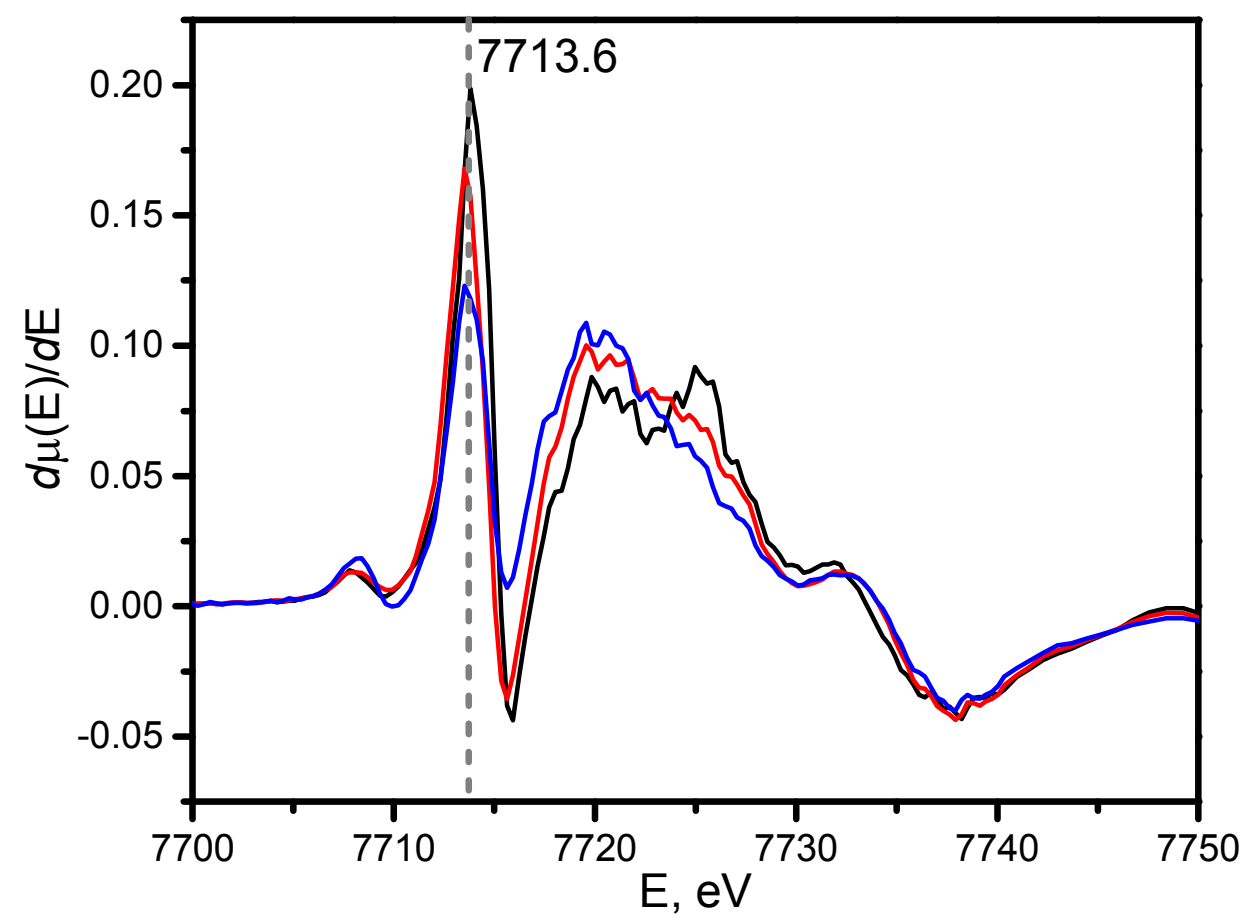

Figure S3. Derivative of XANES spectra for CoTPP (black), Co-PCN-222-act (red), and Co$\mathrm{PCN}-222-\mathrm{O}_{2}$ (blue)

\section{References}

(1). Feng, D.; Gu, Z.; Li, J.; Jiang, H.; Wei, Z.; Zhou, H. Angew. Chem. Int. Ed. 2012, 51, 1030710310 . 